\title{
Aislamiento de Cryptococcus gattii serotipo B a partir de detritos de Eucalyptus spp. en Colombia
}

\author{
Patricia Escandón ${ }^{1}$, Elizabeth Quintero ${ }^{2}$, Diana Granados ${ }^{1}$, Sandra Huérfano ${ }^{1}$, \\ Alejandro Ruiz ${ }^{2}$, Elizabeth Castañeda ${ }^{1}$
}

${ }^{1}$ Grupo de Microbiología, Instituto Nacional de Salud, Bogotá, D. C., Colombia.
${ }^{2}$ Universidad de Puerto Rico, Recinto Universitario de Mayagüez, Puerto Rico.

Introducción. Cryptococcus gattii serotipo B ha sido asociado con diferentes especies de árboles en regiones tropicales y subtropicales. Este serotipo es el segundo de mayor importancia clínica en Colombia, pero no se había recuperado del ambiente en ninguno de los estudios realizados por nuestro grupo.

Objetivo. Para establecer la presencia y distribución de las especies del complejo $C$. neoformans, especialmente del serotipo $\mathrm{B}$, y determinar algunas características fenotípicas relacionadas con la virulencia de los aislamientos recuperados, se diseñó un muestreo de material vegetal en un bosque de piso térmico frío del departamento de Cundinamarca.

Materiales y métodos. Durante febrero y marzo de 2003 se realizaron 4 muestreos en el bosque y se tomaron 167 muestras de detritos de eucaliptos y 28 muestras de otras especies de árboles, las cuales se procesaron empleando el método convencional de extracción y siembra en medio selectivo. Se determinó el tamaño celular y capsular en los filtrados, el crecimiento a $37^{\circ} \mathrm{C}$, la virulencia en un modelo animal y la pareja sexual por PCR.

Resultados. Se aisló C. gattii serotipo B de 46 muestras de eucaliptos (27,5\%). El tamaño celular en el extracto estuvo entre 3,10 y $4,15 \mu \mathrm{m}$ y el tamaño capsular entre 0,30 y 0,46 $\mu \mathrm{m}$. Los 46 aislamientos crecieron a $37^{\circ} \mathrm{C}$, pero ninguno de los dos aislamientos empleados causó la muerte de los animales inoculados durante los 70 días de observación. Todos los aislamientos fueron pareja sexual a.

Conclusiones. Este estudio es el primer informe sobre el aislamiento de C. gattii, serotipo B, pareja sexual a, en Colombia a partir de detritos de eucaliptos.

Palabras clave: Cryptococcus, hábitat, Eucalyptus, fenotipo, virulencia.

Isolation of Cryptococcus gattii serotype B from detritus of Eucalyptus trees in Colombia

Introduction. Cryptococcus gattii serotype B has been associated with several species of trees in tropical and subtropical regions. Serotype $B$ has been found to be the second most frequently isolated from patients in Colombia, but it has not been isolated from the environment. Therefore, a study was designed to sample plant material from a forest in the province of Cundinamarca, with the aim of establishing the presence and distribution of the $C$. neoformans complex, specially serotype B. In addition, isolates were evaluated for several phenotypic characteristics associated with virulence.

Methodology. During February and March, 2003, 4 separate sample collections were made. One hundred sixty-seven samples were collected from detritus of eucalyptus and 28 samples from other species of trees. All were processed using the conventional procedure and plated on selective media. The following parameters were measured: cellular and capsular sizes in the filtrates, growth at $37^{\circ} \mathrm{C}$, virulence in a mouse animal model system, and mating type as detected by PCR.

Results. Cryptococcus gattii serotype B was isolated from 46 eucalyptus samples (27.5\%). The cellular size in the filtrates ranged from 3.10 to $4.15 \mu \mathrm{m}$ and the capsular size from 0.30 and $0.46 \mu \mathrm{m}$. The 46 isolates grew at $37^{\circ} \mathrm{C}$, but none of them caused the death in the animals during 70-day observation period. All isolates were of mating type a. 
Conclusions. This constitutes the first report of the isolation of serotype $B$, mating type $a$, from eucalyptus detritus in Colombia.

Key words: habitat, Cryptococcus, Eucalyptus, phenotype, virulence.

El complejo Cryptococcus neoformans está conformado por cepas patógenas para el hombre y los animales, las que ocasionan la criptococosis, entidad que es causa importante de morbilidad y mortalidad en pacientes inmunosuprimidos e inmunocompetentes (1). La infección es adquirida por la inhalación de los propágulos infectantes presentes en el ambiente y, por esta razón, es de gran importancia el estudio del hábitat de este hongo (1-3). El complejo C. neoformans fue recientemente clasificado en 2 especies y 5 serotipos con base en sus características morfológicas, bioquímicas y genéticas, como sigue: $C$. neoformans variedad grubii, serotipo $A ; C$. neoformans variedad neoformans, serotipo D; C. gattii, serotipos B y C, y el híbrido serotipo AD (4-6).

La variedad grubii tiene una distribución mundial y su hábitat se asocia con excrementos de aves y detritos de diferentes especies de árboles $(3,7,8)$. La variedad neoformans está restringida, especialmente, a países europeos y su hábitat también se ha asociado con excrementos de aves (9). Por otra parte, C. gattii parecía restringido a regiones tropicales y subtropicales; sin embargo, recientemente el serotipo $B$ se asoció con un brote de criptococosis ocurrido en la isla de Vancouver en Canadá, demostrando así la habilidad de esta especie para establecer su hábitat en regiones templadas $(2,10)$. El serotipo B se asocia principalmente con diferentes especies de eucaliptos en Australia, California y Papua, Nueva Guinea $(2,11)$. El serotipo C sólo ha sido recuperado a partir de detritos de almendros (Terminalia cattapa) en Colombia (12) y en Puerto Rico (E. Quintero, comunicación personal).

En Colombia, la frecuencia de los serotipos recuperados de pacientes con criptococosis es

\footnotetext{
Correspondencia:

Patricia Escandón, Grupo de Microbiología, Instituto Nacional de Salud, Avenida Calle 26 \# 51-60, zona 6, CAN, Bogotá, D. C., Colombia.

Teléfono: + (57) 1 220-7700, extensión 445, 446; fax: + (57) 1 220-7700, extensión 445

pescandon@ins.gov.co
}

Recibido: 02/05/05; aceptado: 01/08/05 similar a la informada a nivel mundial: serotipo $A$, $95 \%$; serotipo $B, 4,1 \%$, y serotipos D y C, $0,2 \%$ y $0,8 \%$, respectivamente (Grupo Colombiano de Trabajo en Criptococosis. Abstracts, Sixth International Conference in Cryptococcus and Cryptococcosis, Boston, Massachusetts, June 2428, 2005. Abstract E5). Sin embargo, sólo los serotipos $A$ y $C$ han sido recuperados a partir de fuentes ambientales (12-15).

Por este motivo, se diseñó un estudio con el objeto de determinar la presencia y la distribución de los serotipos del complejo $C$. neoformans en el ambiente, mediante un muestreo amplio en un piso térmico frío. Además, se evaluaron algunas características fenotípicas de los aislamientos recuperados, relacionadas con la virulencia.

Los datos presentados son el resultado de la búsqueda del hongo en un bosque secundario localizado en La Calera, departamento de Cundinamarca (16). Se logró recuperar por primera vez en Colombia aislamientos de $C$. gattii, serotipo $\mathrm{B}$, a partir de detritos de eucaliptos. Además, los estudios de virulencia realizados con dos aislamientos sugieren que éstos pueden ser clasificados como de baja virulencia y, también, que todos pertenecen a la pareja sexual a, constituyéndose este último dato en otro marcador de la baja virulencia de los aislamientos (1).

\section{Materiales y métodos}

\section{Área de estudio}

La Calera es un municipio ubicado en el departamento de Cundinamarca, a 28 kilómetros al noroeste de Bogotá, la capital de Colombia. Este municipio está localizado a una latitud de $4^{\circ} 43$ $\mathrm{N}$ y una longitud de $73^{\circ} 58 \mathrm{O}$ y se caracteriza por tener dos pisos térmicos: frío y templado. La Calera está ubicada a una altitud de 2.718 metros sobre el nivel del mar, su temperatura promedio es de $12,7^{\circ} \mathrm{C}$ y su precipitación anual de $250 \mathrm{~mm}$ (16).

\section{Muestras}

Entre los meses de febrero y marzo de 2003, se realizaron 4 visitas al área, en las cuales se 
recogieron 195 muestras de detritos de árboles. Cuatro de estas muestras de eucaliptos, se tomaron empleando la técnica del hisopo humedecido en solución salina estéril y frotándolo en los detritos (17). Las otras muestras se recogieron con una pala tomando $10 \mathrm{~g}$, aproximadamente, que se colocaron en bolsas plásticas con cierre hermético.

\section{Procesamiento de las muestras}

Las muestras recolectadas con la técnica del hisopo se inocularon directamente en agar Guizotia abyssinica con suplemento de bifenil $(0,1 \%)$, y se sembró una caja por muestra (18). Las muestras recolectadas en bolsas se procesaron de la siguiente manera: se resuspendieron $5 \mathrm{~g}$ de cada una en $25 \mathrm{ml}$ de solución tampón fosfato salino $\mathrm{pH} 7,4$; se mezclaron durante 30 minutos y se filtraron a través de una gasa estéril. Posteriormente, al filtrado se le adicionaron $50 \mu \mathrm{l}$ de una solución con $20 U$ de penicilina y $40 U$ de estreptomicina; se inocularon $100 \mu \mathrm{l}$ de este filtrado en el medio $G$. abyssinica con suplemento de bifenil y antibióticos a las concentraciones ya mencionadas. El medio se incubó a $25^{\circ} \mathrm{C}$ durante 10 días y, posteriormente, se realizó el recuento de las unidades formadoras de colonia por gramo de muestra (UFC/g) (18).

Además, los filtrados se usaron para realizar una observación directa al microscopio, con tinta china, y con el objetivo de 40X se determinó la presencia de células capsuladas. En dos de los filtrados que fueron positivos por observación directa se determinó, también, el tamaño celular y capsular de las levaduras; la lectura del diámetro se realizó con el empleo de un micrómetro ocular con resolución de $1 \mu \mathrm{m}$ y con el objetivo 100X. En cada uno de los filtrados se realizaron 20 mediciones del tamaño celular y capsular, las cuales fueron promediadas (19). El pH de las muestras recolectadas en bolsas se determinó resuspendiendo $1 \mathrm{~g}$ de cada muestra en $10 \mathrm{ml}$ de solución de $\mathrm{CaCl}_{2}(0,1 \mathrm{M})(20)$.

\section{Identificación}

Las colonias que mostraran color café en el medio de $G$. abyssinica por la acción de la enzima fenol oxidasa sobre el sustrato, se inocularon en agar glucosado de Sabouraud. Posteriormente, se realizó la observación microscópica en preparaciones con agua destilada y tinta china (19). También se evaluó la producción de ureasa (21), se determinó la variedad empleando el medio canavanina, glicina, azul de bromotimol (CGB) (22) y el serotipo mediante aglutinación con anticuerpos policlonales específicos para el polisacárido capsular (Crypto-Check, Iatron Laboratories, Tokyo, Japan). Los aislamientos recuperados se conservaron a $-70^{\circ} \mathrm{C}$ en glicerol al 10\% en la colección del Grupo de Microbiología.

\section{Características fenotípicas asociadas con la virulencia}

Se evaluó el crecimiento de los aislamientos en agar glucosado de Sabouraud a $37^{\circ} \mathrm{C}$ (19) y se estudió la virulencia en un modelo animal (23). Se usaron ratones BALB/C machos de 3 a 5 semanas, obtenidos en el bioterio del INS. Durante el estudio se mantuvieron en la sala de alta seguridad del bioterio, con una humedad relativa entre $40 \%$ y $60 \%$ y una temperatura entre 18 y $22^{\circ} \mathrm{C}$. El manejo de los animales se realizó de acuerdo con las recomendaciones nacionales (Ley 84 de 1989, Resolución 8430 de 1993), internacionales (Consejo de Comunidades Europeas y del Concilio Canadiense sobre el cuidado de animales de 1998) y el protocolo de uso de animales del INS. Se emplearon 5 ratones BALB/c para cada uno de los dos aislamientos evaluados, recuperados de las muestras que presentaron el mayor número de UFC/g de muestra (INS H0058-1690 e INS H0058-1706) y dos controles: INS H0058-579 (serotipo A), que causa la muerte de los animales en 2 semanas, aproximadamente, e INS H0058-1320 (serotipo B) que no causa la muerte en los animales durante el período de observación establecido en este estudio (24). Cada animal fue inoculado con $5 \times 10^{5}$ UFC del aislamiento en la vena lateral de la cola, con observación diaria durante 70 días; a los animales que murieron se les practicó la autopsia, al igual que a los que se encontraron adinámicos o erizados, previa eutanasia con $\mathrm{CO}_{2}$. Los sobrevivientes se sacrificaron el día 70 posinoculación, empleando eutanasia con $\mathrm{CO}_{2}$. Se obtuvieron el cerebro, el bazo y el pulmón de cada animal, los cuales se procesaron para determinar 
el número de UFC/g de órgano en agar glucosado de Sabouraud $(23,24)$.

\section{Pareja sexual}

Se determinó la pareja sexual mediante PCR con iniciadores específicos diseñados para amplificar una región del gen de la feromona $\alpha$ con un tamaño de 109 pb (25) y el gen de la feromona a con un tamaño de 213 pb (26). Las secuencias de los iniciadores empleados fueron:

MF $\alpha \cup$ 5'TTCACTGCCATCTTCACCACC3', MF $\alpha$ L 5'TCTAGGCGATGACACAAAGGG3', MFa2U 5'ACACCGCCTG TTACAATGGAC3' y MFa2L 5'CAGCGTTTTGAAGATGGACTTT3'.

Primero se realizó la PCR para la amplificación del gen de la feromona $\alpha$ y en aquellos aislamientos que no amplificaron se realizó la PCR para amplificar el gen de la feromona a. Las condiciones de la PCR para la amplificación del gen de la feromona $\alpha$, para un volumen final de 50 $\mu$, fueron: $1 X$ de tampón de PCR, $200 \mathrm{mM}$ de cada dNTP, $15 \mathrm{mM}$ de $\mathrm{MgCl}_{2}, 0,2 \mu \mathrm{m}$ de cada iniciador, 2,5 $U$ de Taq polimerasa (Invitrogen) y $1 \mu \mathrm{l}$ de ADN (extraído por la técnica de ebullición o por la técnica de extracción con perlas de vidrio) $(25,27,28)$. Para la amplificación del gen de la feromona a se emplearon las condiciones anteriormente descritas, excepto que la concentración de los iniciadores fue de $50 \mathrm{ng} / \mathrm{ml}$ (26). Se emplearon como cepas control los aislamientos INS H0058-1962 (CBS 884), serotipo A, pareja sexual $\alpha$ e INS H0058-1971 (CBS 6998), serotipo B, pareja sexual a (suministrados por Dee Carter de la Universidad de Sydney, Australia).

Los productos de amplificación se separaron en geles de agarosa al 2\% durante 1 hora a 100 voltios y se visualizaron en un transiluminador de luz ultravioleta para la posterior toma de la fotografía.

\section{Resultados}

\section{Recuperación de Cryptococcus gattii}

Entre febrero 6 y marzo 12 se obtuvieron 167 muestras de Eucalyptus spp. y 28 de otras especies de árboles que se encontraban en la zona, incluidos pinos (Pinus sp.), árbol blanco de raíz de serpiente (Ageratina altissima), laurel (Laurus nobilis), raque (Vallea stipularis) y guaba
(Phytolacca bogotensis). De algunos de los árboles se obtuvieron muestras en más de una ocasión. C. gattii, serotipo B se recuperó a partir de $46(27,5 \%)$ de las 167 muestras de eucaliptos procesadas. Dos de las muestras positivas se recuperaron por la técnica del hisopo humedecido en solución salina estéril. El porcentaje de muestras positivas en las diferentes fechas fue: febrero $6,3 / 40(7,5 \%)$, marzo $6,32 / 40(80 \%)$ y marzo 12, 11/87 (12,5\%). El hongo no se aisló de las muestras recolectadas a partir de otras especies de árboles. La temperatura ambiental promedio en el sitio de muestreo, en los meses de febrero y marzo, estuvo entre 12,8 y $13,1^{\circ} \mathrm{C}$.

La densidad de la población de $C$. gattii en las muestras positivas estuvo entre 50 y $15 \times 10^{6} \mathrm{UFCl}$ g (cuadro 1). La observación microscópica realizada en los filtrados de las 46 muestras de las que se había recuperado el hongo, confirmó la presencia de levaduras con cápsulas pequeñas en $30(65,2 \%)$ de éstas. Dos de los filtrados que presentaron el mayor número de levaduras en la preparación con tinta china $\left(15 \times 10^{3}-15 \times 10^{6}\right.$ UFCl $\mathrm{g})$, se seleccionaron para determinar el tamaño celular y capsular de las mismas. El tamaño celular en los dos filtrados estuvo entre $3,10 \pm 0,83 \mu \mathrm{m}$ y $4,15 \pm 0,67 \mu \mathrm{m}$ y el tamaño capsular entre $0,46 \pm 0,29 \mu \mathrm{m}$ y $0,30 \pm 0,10 \mu \mathrm{m}$, respectivamente.

Cuadro 1. Observación microscópica de las muestras positivas y densidad de la población (UFC/g) de Cryptococcus gattii.

\begin{tabular}{ccc}
\hline $\begin{array}{c}\text { Muestras } \\
\mathbf{n = 4 6}\end{array}$ & Levaduras/campo $^{\text {a }}$ & UFC/g de muestra \\
\hline 5 & 0 & 50 \\
3 & $0-1$ & 50 \\
4 & 0 & $150-200$ \\
5 & 0 & $400-550$ \\
9 & $0-1$ & $400-550$ \\
1 & 0 & $750-900$ \\
2 & $0-1$ & $750-900$ \\
1 & 0 & $1,3-1,7 \times 10^{3}$ \\
3 & $0-1$ & $1,3-1,7 \times 10^{3}$ \\
4 & $0-1$ & $2,5-2,9 \times 10^{3}$ \\
1 & $0-1$ & $5 \times 10^{3}$ \\
6 & $0-1$ & $15-20 \times 10^{3}$ \\
$1^{\mathrm{b}}$ & 3 & $15-20 \times 10^{3}$ \\
$1^{\mathrm{b}}$ & $>10$ & $15 \times 10^{6}$ \\
\hline
\end{tabular}

\footnotetext{
a Observación microscópica realizada con tinta china, $40 \mathrm{X}$ ${ }^{b}$ Muestras seleccionadas para determinar tamaño celular y capsular
} 
El pH de las muestras osciló entre 3,3 y 7,2, tanto en las muestras positivas como en las negativas.

\section{Características fenotípicas asociadas con la virulencia}

Los 46 aislamientos de C. gattii crecieron a $37^{\circ} \mathrm{C}$. Los dos aislamientos seleccionados para evaluar la virulencia en el modelo animal no fueron letales para los animales durante el período de observación de 70 días; sin embargo, en la autopsia realizada se observó la aparición de criptococomas en el pulmón y la diseminación del hongo a cerebro, bazo y pulmón, como se observa en la figura 1. Los 5 animales inoculados con el aislamiento control serotipo A presentaron síntomas de letargia 12 días posinoculación, por lo que fueron sacrificados inmediatamente; en la autopsia se observó la diseminación del hongo a cerebro, pulmones y bazo (figura 1). En cuanto a los animales inoculados con el serotipo $B$, en la autopsia a los 70 días se observó la aparición de hidrocefalia y diseminación a cerebro, bazo y pulmón (figura 1).

\section{Determinación de la pareja sexual}

Todos los aislamientos amplificaron para el gen de la pareja sexual a, y generaron un fragmento

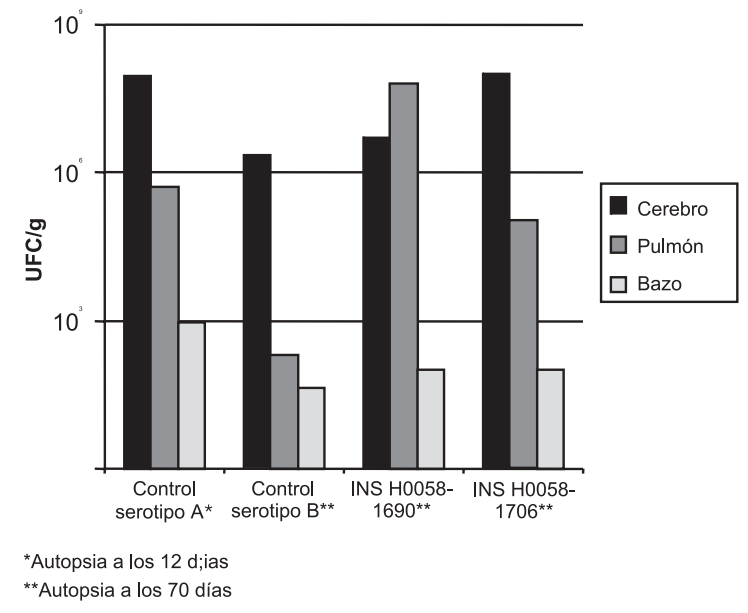

Figura 1. Unidades formadoras de colonia (UFC) recuperadas a partir de los órganos de los animales inoculados en la vena lateral de la cola con $5 \times 10^{5}$ UFC de los aislamientos ambientales de C. gattii, serotipo B (INS1690, INS-1706) y de los aislamientos control de $C$. neoformans var. grubii, serotipo A y C. gattii, serotipo B.

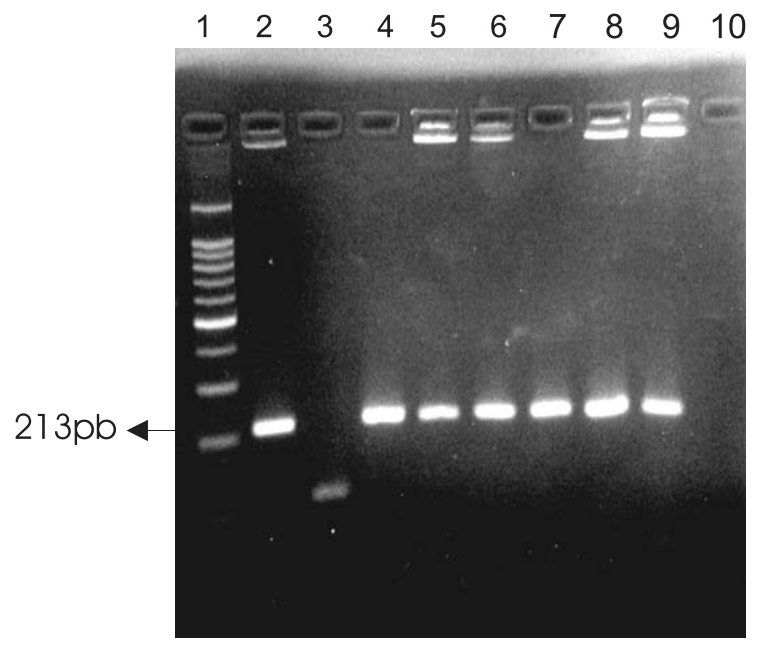

Figura 2. Gel representativo de la amplificación por PCR del gen de la pareja sexual de aislamientos ambientales de Cryptococcus gattii, serotipo B, con los iniciadores MFa2U y MFa2L.

Carril 1: marcador de peso molecular de $100 \mathrm{pb}$; carril 2: cepa control INS H0058-1971 (Cryptococcus gattii, serotipo B, pareja sexual a); carril 3: cepa control INS H0058-1962 (Cryptococcus gattii, serotipo B, pareja sexual a); carriles 4-9: aislamientos ambientales Cryptococcus gattii, serotipo B (INS H0058-1689 - INS H0058-1694); carril 10: control negativo.

de $213 \mathrm{pb}$, como se observa en la figura 2, en la cual, igualmente, se ve la amplificación del ADN de la cepa INS H0058 1971 (CBS 6998), serotipo $B$, pareja sexual a, empleada como control. En ninguno de los aislamientos se obtuvo amplificación con los iniciadores específicos para la pareja sexual $\alpha$.

\section{Discusión}

El presente estudio se destaca como el primer informe acerca del aislamiento de $C$. gattii, serotipo $B$, pareja sexual a, a partir de detritos de eucaliptos en Colombia.

Actualmente, existen muchos interrogantes en cuanto a la ecología del complejo C. neoformans, especialmente en cuanto a su ciclo de vida. Ellis, basado en sus hallazgos (2), propuso en 1990 que los eucaliptos eran el hábitat primario de $C$. gattii, serotipo B; sin embargo, estudios posteriores indicaron que los eucaliptos no eran el único nicho ambiental del hongo, que la madera en descomposición podría servir como substrato para 
que se lleve a cabo una propagación clonal y que el nicho ecológico primario pudiera estar asociado con otro tipo de material vegetal (29). Es así como en Brasil se ha reportado el aislamiento del serotipo $\mathrm{B}$ de detritos de árboles como Moquilea tomentosa (30) y en Colombia se publicó el aislamiento del serotipo $\mathrm{C}$ a partir de detritos de almendros (Terminalia cattapa) en Cúcuta, Norte de Santander (12). Así mismo, se ha logrado recuperar el serotipo $B$ a partir de árboles diferentes a los eucaliptos en Buenos Aires, Argentina (31). Los aislamientos responsables del brote en la isla de Vancouver, Canadá fueron recuperados a partir del enebro (Juniperus virginiana), del madroño (Arbutus spp.) y del arce (Acer sp.), lo cual sugiere que este serotipo también se puede encontrar asociado con otras especies de árboles (10). Por lo tanto, la frecuente recuperación del hongo a partir de detritos sugiere que los árboles son un elemento importante en el ciclo de vida de C. gattii (32), lo que se podría explicar por su capacidad de producir lacasa, enzima involucrada en el proceso de la degradación de la lignina, y que la madera en descomposición sea un substrato adecuado para el crecimiento del hongo $(1,2,8,29,32)$. Nuestros resultados sugieren que $C$. gattii puede, efectivamente, tener una asociación con los eucaliptos, y los convirtierta en un nicho ecológico apropiado para su supervivencia en el ambiente colombiano.

Por otra parte, el pequeño tamaño de las levaduras $(3,10$ a $4,15 \mu \mathrm{m})$ en las muestras que permitieron la recuperación del hongo en este estudio, tiene gran importancia debido a que los propágulos de menor tamaño $(<3 \mu \mathrm{m})$ pueden llegar hasta los alvéolos pulmonares e iniciar la infección en el humano. Lo anterior parece indicar que estos árboles representan un reservorio que sirve como foco de infección para el hombre y los animales $(1,29,33)$.

Los resultados obtenidos en relación a la medición del $\mathrm{pH}$ de las muestras sugieren que esta variable no es un determinante del hábitat del hongo, debido al amplio rango de $\mathrm{pH}$ que presentaron las muestras $(3,3$ a 7,2$)$.

En cuanto a la virulencia, se sabe que el crecimiento a $37^{\circ} \mathrm{C}$ es una condición básica para la patogenicidad $(1,34)$. Los aislamientos recuperados en este estudio mostraron esta característica. Los dos aislamientos serotipo B recuperados y utilizados en la evaluación de la virulencia en el modelo animal no produjeron la muerte de los animales durante el período de observación, lo cual concuerda con estudios realizados previamente donde se demostró que la virulencia es menor para C. gattii que para $C$. neoformans var. grubii $(33,34)$.

La pareja sexual de los aislamientos del complejo C. neoformans ha sido tradicionalmente determinada mediante apareamientos in vitro con cepas control en medios de cultivo pobres en fuentes de nitrógeno (1); sin embargo, este método presenta algunos problemas ya que el apareamiento es sensible a una gran variedad de condiciones ambientales como temperatura, disponibilidad de nutrientes y humedad $(1,35)$. Por lo tanto, se han desarrollado nuevos métodos para determinar los alelos de la pareja sexual de este hongo, como la PCR con iniciadores específicos empleada en este estudio. La amplificación del gen de la pareja sexual a en todos los aislamientos pudiera indicar la presencia de una población clonal, sugiriendo que esta clonalidad puede influenciar, o ser influenciada, por la ausencia de la pareja sexual opuesta en la naturaleza y limitar así su recombinación $(36,37)$. Se ha encontrado que la prevalencia de la pareja sexual $\alpha$ sobre la pareja sexual a se debe a la gran capacidad de la primera de llevar a cabo el proceso de fructificación haploide en ausencia de la pareja sexual opuesta $(1,38,39)$. Sin embargo, esta prevalencia de la pareja sexual $\alpha$ sobre la a varía según lo han reportado diversos autores en varias regiones australianas; en Adelaida, al sur de Australia, se ha encontrado una proporción $\alpha: a$ de 26:0; en Queensland (sureste de Australia), 15:0; en New South Wales 2:27, lo cual sugiere en todos ellos que se está llevando a cabo una reproducción clonal; mientras que en Renmark (noroeste de Australia), la proporción fue 10:6, lo que indica la posibilidad de que se esté presentando recombinación sexual entre los aislamientos (37). El hallazgo de la pareja sexual a en todos los aislamientos recuperados en el presente estudio difiere de lo encontrado en los 
aislamientos del brote de criptococosis en Vancouver, los cuales correspondieron todos a la pareja sexual $\alpha$ (40), por lo que sería de gran interés realizar estudios colaborativos que nos permitieran comparar fenotípica y genotípicamente estos dos grupos de aislamientos.

Los estudios posteriores realizados por el Grupo de Microbiología, que no han sido publicados todavía, han demostrado que todos los aislamientos de $C$. gattii, serotipo $B$, recuperados en La Calera, al ser tipificados molecularmente mediante PCR huella digital y RFLP, pertenecen al patrón molecular VGII, lo cual sugiere nuevamente la presencia de una población clonal, y se relaciona con el hecho de que todos los aislamientos causantes del brote en la isla de Vancouver, Canadá, también fueron agrupados dentro de este mismo patrón molecular (40).

Este hallazgo constituye el primer informe del aislamiento de $C$. gattii, serotipo $B$, a partir de fuentes ambientales en Colombia y, por lo tanto, es la primera vez que se obtienen datos en cuanto a sus características fenotípicas como el tamaño celular y capsular en la naturaleza, el crecimiento a $37^{\circ} \mathrm{C}$ y la pareja sexual, características que, aparentemente, están asociadas con la baja virulencia de los aislamientos evaluados en el modelo animal. Estos hallazgos corresponden con lo informado en la literatura y señalan el desconocimiento que tenemos sobre la patogénesis de la criptococosis ocasionada por esta especie y serotipo y que se manifiesta en el hospedero inmunocompetente.

\section{Financiación}

Del Instituto Colombiano para el Desarrollo de la Ciencia y la Tecnología "Francisco José de Caldas", Colciencias (código 2104-04-11802), el Instituto Nacional de Salud y la Universidad de Puerto Rico.

\section{Conflictos de intereses}

Los autores declaran que no existe conflicto de interés en esta publicación.

\section{Referencias}

1. Casadevall A, Perfect JR. Cryptococcus neoformans. Washington, D. C.: American Society for Microbiology Press; 1998. p.541.
2. Ellis DH, Pfeiffer TJ. Natural habitat of Cryptococcus neoformans var. gattii. J Clin Microbiol 1990;28:1642-4.

3. Sorrel TC, Ellis DH. Ecology of Cryptococcus neoformans. Rev Iberoam Micol 1997;14:42-3.

4. Franzot S, Salkin I, Casadevall A. Cryptococcus neoformans var. grubii: separate varietal status for Cryptococcus neoformans serotype A isolates. J Clin Microbiol 1999;37:838-40.

5. Boekhout T, Theelen B, Diaz M, Fell J, Hop W, Abeln E et al. Hybrid genotypes in the pathogenic yeast Cryptococcus neoformans. Microbiology 2001;147: 891-907.

6. Kwon-Chung K, Boekhout T, Fell J, Diaz M. Proposal to conserve the name Cryptococcus gattii against $C$. hondurianus and C. bacillisporus (Basidiomycota, Hymenomycetes, Tremellomycetidae). Taxon 2002;51: 804-6.

7. Emmons C. Isolation of Cryptococcus neoformans from soil. J Bacteriol 1951;62:685-9.

8. Lazera MS, Pires FDA, Coura C, Nishikawa MM, Bezerra CCF, Trilles L et al. Natural habitat of Cryptococcus neoformans in decaying wood forming hollows in living trees. J Med Vet Mycol 1996;34:127-31.

9. Garcia-Hermoso D, Mathoulin-Pelissier S, Couprie B, Ronin O, Dupont B, Dromer F. DNA typing suggest pigeon droppings as a source of pathogenic Cryptococcus neoformans serotype D. J Clin Microbiol $1997 ; 35: 2683-5$

10. Stephen C, Lester S, Black W, Fyfe M, Raverty S. Multispecies outbreak of cryptococcosis on southern Vancouver Island, British Columbia. Can Vet J 2002; 43:792-4.

11. Sorrell T. Cryptococcus neoformans variety gattii. Med Mycol 2001;39:155-68.

12. Callejas A, Ordoñez N, Rodriguez MC, Castañeda E. First isolation of Cryptococcus neoformans var. gattii serotype $C$, from the environment in Colombia. Med Mycol 1998;36:341-4.

13. Quintero E, Castañeda E, Ruiz A. Distribución ambiental de Cryptococcus neoformans en el departamento de Cundinamarca, Colombia. Rev Iberoam Micol 2005;22:93-8.

14. Caicedo LD, Alvarez MI, Delgado M, Cárdenas A. Cryptococcus neoformans in bird excreta in the city zoo of Cali, Colombia. Mycopathologia 1991;147:121-4.

15. Duarte A, Ordóñez N, Castañeda E. Asociación de levaduras del género Cryptococcus con especies de Eucalyptus en Santafé de Bogotá. Rev Inst Med Trop Sao Paulo 1994;36:125-30.

16. Instituto Geográfico Agustín Codazzi, Subdirección de Investigación y Divulgación Geográfica. Diccionario Geográfico de Colombia. 
Segunda edición. Bogotá, D. C.: Instituto Geográfico Agustín Codazzi; 1980. p.829.

17. Krockenberger M, Canfield P, Malik R. Cryptococcus neoformans in the koala (Phascolarctos cinereus): colonisation by $C$. $n$. var. gattii and investigation of environmental sources. Med Mycol 2002;40:263-72.

18. Staib F. Sampling and isolation of Cryptococcus neoformans from indoor air with the aids of the Reuter centrifugal sampler (RCS) and Guizotia abyssinica creatinine agar. A contribution to the mycologicalepidemiological control of $\mathrm{Cr}$. neoformans in the fecal matter of caged birds. Zentralbl Bakteriol Mikrobiol Hyg 1985;180:567-75.

19. Franzot SP, Mukherjee J, Cherniak R, Chen LC, Hamdan JS, Casadevall A. Microevolution of a standard strain of Cryptococcus neoformans resulting in differences in virulence and other phenotypes. Infect Immunol 1998;66:89-97.

20. Shinner F, Ohlinger R, Kandeler E, Masgesin R. Methods in soil biology. XI edición. Berlin, Germany: Spring Verlag Eds.; 1996. p.426.

21. Kwon-Chung KJ, Wickes BL, Booth JL, Vishniac HS, Bennet JE. Urease inhibition by EDTA in the two varieties of Cryptococcus neoformans. Infect Immun 1987;55:1751-4.

22. Kwon-Chung KJ, Polacheck I, Bennet J. Improved diagnostic medium for separation of Cryptococcus neoformans var. neoformans (serotypes $A$ and $D$ ) and Cryptococcus neoformans var. gattii (serotypes B and C). J Clin Microbiol 1982;15:535-7.

23. Fries B, Taborda CP, Serfass E, Casadevall A. Phenotypic switching of Cryptococcus neoformans occurs in vivo and influences the outcome of infection. J Clin Invest 2001;108:1639-48.

24. Huérfano S, Cepero MC, Castañeda E. Caracterización fenotípica de aislamientos ambientales de Cryptococcus neoformans. Biomédica 2003;23:328-40.

25. Halliday CL, Carter DA. Clonal reproduction and limited dispersal in an environmental population of Cryptococcus neoformans var. gattii isolates from Australia. J Clin Microbiol 2003;41:703-11.

26. Fraser J, Subaran L, Nichols C, Heitman J. Recapitulation of the sexual cycle of the primary fungal pathogen Cryptococcus neoformans var. gattii: implications for an outbreak on Vancouver Island, Canada. Eukaryot Cell 2003;2:1036-45.

27. Chaturvedi S, Rodeghier B, Fan J, McClelland C, Wickes B, Chaturvedi V. Direct PCR for Cryptococcus neoformans MAT $\alpha$ and MATa pheromones to determine mating type, ploidy, and variety: a tool for epidemiological and molecular pathogenesis studies. J Clin Microbiol 2000;38:2007-9.

28. Casali A, Goulart L, Rosa e Silva L, Ribeiro A, Amaral A, Alves $S$ et al. Molecular typing of clinical and environmental Cryptococcus neoformans isolates in the Brazilian state of Rio Grande do Sul. FEMS Yeast Res 2003;3:405-15.

29. Cabral LF. Wood, animals and human beings as reservoirs for human Cryptococcus neoformans infection. Rev Iberoam Micol 1999;16:77-81.

30. Lazera M, Wanke B, Nishikawa M. Isolation of both varieties of Cryptococcus neoformans from saprophytic sources in the city of Rio de Janeiro, Brazil. J Med Vet Mycol 1993;31:449-54.

31. Davel G, Abrantes R, Brudny M, Cordoba S, Rodero L, Canteros CE et al. 1st environmental isolation of Cryptococcus neoformans var. gattii in Argentina. Rev Argent Microbiol 2003;35:110-2.

32. Sorrell T, Brownlee A, Ruma P, Malik R, Pfeiffer T, Ellis DH. Natural environmental sources of Cryptococcus neoformans var. gattii. J Clin Microbiol 1996;34:1261-3.

33. Buchanan KL, Murphy JW. What makes Cryptococcus neoformans a pathogen? Emerg Infect Dis 1998;4:71-83.

34. Castañeda E. En búsqueda del hábitat de Cryptococcus neoformans var. gattii en Colombia. Rev Acad Colomb Ciencias 2001;25:105-14.

35. Yan Z, Li X, Xu J. Geographic distribution of mating type alleles of Cryptococcus neoformans in four areas of the United States. J Clin Microbiol 2002;40:965-72.

36. Kwon Chung KJ, Edman J, Wickes B. Genetic association of mating types and virulence in Cryptococcus neoformans. Infect Immun 1992;60: 602-5.

37. Halliday C, Bui T, Krockenberger M, Malik R, Ellis D, Carter D. Presence of $\alpha$ and a mating types in environmental and clinical collections of Cryptococcus neoformans var. gattii strains from Australia. J Clin Microbiol 1999;37:2920-6.

38. Wickes B, Mayorga M, Edman U, Edman J. Dimorphism and haploid fruiting in Cryptococcus neoformans: association with the $\alpha$-mating type. Proc Natl Acad Sci USA 1996;93:7327-31.

39. Tscharke R, Lazera M, Chang $Y$, Wickes B, Kwon Chung K. Haploid fruiting in Cryptococcus neoformans is not mating type alpha-specific. Fungal Genet Biol 2003;39:230-7. 